\title{
Aerolíneas bajo costo y convencionales: la satisfacción y lealtad de sus clientes
}

\author{
Juan Carlos Garmendia Mora* \\ Diego Alejandro Tavera Morales**
}

Fecha de recibido: 23 de abril de 2021

Fecha de aprobado: 7 de octubre de 2021

Para citar este artículo: Garmendia Mora, J. C., \& Tavera Morales, D. A. (2021) Aerolíneas bajo costo y convencionales: la satisfacción y lealtad de sus clientes. Revista Universidad E Empresa, 23(41), 1-26. https://doi.org/10.12804/revistas.urosario.edu.co/ empresa/a.10523

\section{Resumen}

Se evaluaron niveles de satisfacción y lealtad del cliente en las aerolíneas de bajo costo y convencionales en Colombia. El enfoque fue cuantitativo, se aplicó una encuesta a 421 usuarios en el aeropuerto El Dorado, de Bogotá. La brecha que separa a estos tipos de aerolíneas es cada vez más reducida, aunque sigue existiendo un diferencial entre la satisfacción que expresan los clientes; el modelo de bajo costo hace que sus pasajeros estén menos satisfechos y, por lo tanto, sean menos leales. Existe una alta correlación entre satisfacción y lealtad: 0.81 para las tradicionales y 0.85 para las de bajo costo.

Palabras clave: aerolíneas bajo costo; aerolíneas tradicionales; satisfacción; lealtad.

* Doctor en Ciencias Gerenciales, Ingeniero Aeronáutico. Profesor de la Institución Universitaria Politécnico Grancolombiano (Bogotá). Correo electrónico: juangarmendia@yahoo.com ORCID: https://orcid.org/0000-0003-0127-4292

*** Tecnólogo en Gestión de Servicios para Aerolíneas, Institución Universitaria Politécnico Grancolombiano (Bogotá). Correo electrónico: ditavera@poligran.edu.co 


\title{
Low-Cost and Conventional Airlines: Customer Satisfaction and Loyalty
}

\begin{abstract}
The present article exposes the evaluation of customer satisfaction and loyalty levels in low-cost and conventional airlines in Colombia. The approach was quantitative; a survey was applied to 421 users at El Dorado airport, Bogota. The gap that separates these types of airlines is becoming smaller, although there is still a differential between customers' expressed satisfaction. The low-cost model makes its passengers less satisfied and, therefore, less loyal. There is a high correlation between satisfaction and loyalty: 0.81 for traditional airlines and 0.85 for low-cost airlines.
\end{abstract}

Keywords: Low-cost airlines; traditional airlines; satisfaction; loyalty.

Companhias aéreas de baixo custo e convencionais: satisfação e fidelidade do cliente

\section{Resumo}

Os níveis de satisfação e fidelidade do cliente foram avaliados em companhias aéreas convencionais e de baixo custo na Colômbia. A abordagem foi quantitativa, uma pesquisa foi aplicada a 421 usuários no aeroporto El Dorado, em Bogotá. A brecha que separa estes tipos de companhias aéreas é cada vez mais estreita, embora ainda exista um diferencial entre a satisfação expressa pelos clientes; o modelo de baixo custo deixa seus passageiros menos satisfeitos e, portanto, menos fiéis. Existe uma alta correlação entre satisfação e lealdade: 0,81 para os tradicionais e 0,85 para os de baixo custo.

Palavras-chave: companhias aéreas de baixo custo; companhias aéreas tradicionais; satisfação; lealdade.

\section{Introducción}

Las líneas aéreas son empresas que se consagran al transporte de pasajeros y carga mediante el uso de aeronaves. Estas han tenido un importante desarrollo desde principios del siglo xx hasta el tiempo presente, en el que su modelo de negocio ha ido evolucionando de acuerdo con la realidad cambiante del mercado. Durante muchos años a lo largo de esa historia, el transporte aéreo de pasajeros fue bastante elitista, debido a los precios de los pasajes, inaccesible para la mayoría de las personas. La incursión de las aerolíneas low cost (bajo costo) en el transporte aéreo generó cambios importantes en el sector; desde entonces, viajar ha sido más fácil para personas con todo tipo de presupuestos, lo que también fue un llamado para las compañías aéreas tradicionales, las cuales tuvieron que hacer modificaciones en su modelo de trabajo (Rodríguez, 2018). 
Las low cost representan una amenaza, desde el punto de vista estratégico, para las aerolíneas convencionales o legacy, dados los mayores costos de operación de estas últimas, lo cual las hace menos idóneas para competir en precios, siendo este un elemento significativo para la generalidad de los pasajeros. Sánchez (2009) afirma que a principios del siglo xxı, años en que el transporte aéreo fue duramente golpeado por el terrorismo, precios altos del petróleo, epidemias como el sARs, la gran mayoría de las líneas aéreas convencionales tuvieron fuertes pérdidas, mientras las compañías low cost estuvieron en positivo, gracias a su modelo de negocio; esta afectación puede estar sucediendo de manera similar a causa de la pandemia del Covid-19 (Barría, 2020). Por ejemplo, en el desarrollo de la pandemia por Covid-19, en muchos países prohibieron el despacho de alimentos dentro del avión, así como el uso de los implementos de entretenimiento en vuelo, dos servicios que constituyen parte importante de la diferencia entre las aerolíneas tradicionales y las de bajo costo, por lo tanto, se ve afectado el modelo de negocios de las tradicionales.

También es necesario indicar que un importante número de líneas aéreas legacy lanzaron sus propias low cost, o han ido modificando el abanico de sus servicios hasta tener una oferta híbrida. Independientemente del tipo de aerolínea, cada pasajero hace su evaluación de la experiencia que tuvo, con lo cual él mismo determinará su grado de satisfacción. El propósito de este artículo es analizar qué tanta satisfacción ha generado cada una de ellas a los pasajeros. Sin embargo, cuando se refiere al término de bajo costo, se sabe que existe una diferencia entre el servicio que brinda esa aerolínea, desde la atención al cliente en el vuelo hasta los beneficios que pueden llegar a ofrecer, y una aerolínea convencional, cuyo propósito es servir al cliente de una forma más completa.

Desde finales del siglo xx se han desarrollado distintas guerras entre las aerolíneas de bajo costo y las tradicionales, esto, por supuesto, con la intención de tomar mayor parte del mercado. En algunos momentos las cifras han favorecido a las low cost, en otros han salido beneficiadas las tradicionales (Molina, 2019). Relacionado con lo anterior, Plummer (2015) indica que en Europa y Estados Unidos esta guerra entre aerolíneas se circunscribía en un principio solo a tratar de establecer el mejor precio para los pasajeros, pero más recientemente las aerolíneas tradicionales de Estados Unidos se dieron cuenta de los beneficios que esto traía a las low cost, por eso, han modificado sus estrategias, realizando acciones tales como la compra de aerolíneas de bajo costo, la mejora en la eficiencia en 
la prestación de los servicios preestablecidos, así como la inclusión de nuevos servicios para tener mayor diferenciación.

En Colombia, el transporte aéreo de pasajeros ha estado durante muchos años en auge, por lo menos hasta 2019 antes de la pandemia. Esto, dentro de otros factores, gracias a la incorporación en la oferta de compañías de bajo costo que han permitido expandir el tamaño del mercado, otorgándole dinamismo, creando opciones diferentes para los pasajeros y prácticamente obligando, como sucedió en otras latitudes, a las aerolíneas tradicionales a modificar sus prácticas y prestaciones de servicio. Dice García (2016) que en el mercado colombiano ha habido muchos movimientos, incursión de nuevas aerolíneas, modificaciones de modelos de negocios hacia un estado híbrido; aerolíneas tradicionales como Avianca y Latam están compitiendo con otras más nuevas, como Viva Colombia, que desde su creación poseen un modelo low cost.

El objetivo general de la investigación fue evaluar los niveles de satisfacción y lealtad de los clientes respecto al servicio prestado por las aerolíneas de bajo costo y las aerolíneas convencionales. La relevancia de esta investigación radica en la necesidad de obtener información de manera científica en cuanto a las variables satisfacción y lealtad en el mercado del transporte doméstico colombiano, la cual puede servir como guía para los futuros usuarios, así como una referencia para las propias aerolíneas sobre el trabajo que están realizando.

\section{Revisión bibliográfica}

\subsection{Antecedentes}

Existe un trabajo de Forgas et al. (2011) denominado "La formación de lealtad de un cliente de una compañía aérea: diferencias entre aerolíneas tradicionales y de bajo coste”, en el que se analizó la lealtad de los usuarios, tomando como principal fuente de información las entrevistas hechas a usuarios de las empresas British Airways y easyJet que viajaban en la ruta Barcelona-Londres. Dentro de los resultados obtenidos destaca que la satisfacción, 
la confianza y el valor percibido explican la lealtad de los clientes hacia las aerolíneas, y, de acuerdo con el tipo de modelo, se producen efectos moderadores.

En los trabajos investigativos relacionados se encuentra el de Pastrana-Martínez et al. (2020) titulado "Análisis de la satisfacción de pasajeros de aerolíneas de bajo costo mexicanas", cuyo objetivo fue indagar sobre la satisfacción como variable mediadora entre la capacidad de respuesta y la confiabilidad, y entre la lealtad y las intenciones de comportamiento; la investigación fue cuantitativa, no experimental, transversal, descriptiva y correlacional. Se hallaron tres relaciones significativas entre las variables estudiadas: confiabilidad y satisfacción, satisfacción y lealtad, y entre satisfacción e intenciones de comportamiento. Esta investigación trabaja dos de las variables que se revisan en el presente documento.

También es esencial mencionar el trabajo de Garmendia (2019) "Satisfacción y lealtad del cliente en las operaciones domésticas de las aerolíneas colombianas". Esta investigación revisa el constructo de servicio al cliente y evalúa los niveles de satisfacción y lealtad,

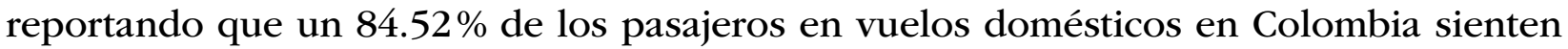
satisfacción por el servicio. Se demuestra igualmente que los programas de fidelización cumplen su propósito, ya que los registros de satisfacción de los clientes leales, así como su intención de volver a usar el servicio, son bastante superiores a los del resto de pasajeros.

\subsection{El servicio al cliente}

El servicio al cliente se remonta a los orígenes del comercio. Dicen Arif et al. (2013) que el servicio al cliente es uno de los factores clave para que cualquier empresa pueda generar ventas y obtener unos niveles de ganancias adecuadas. Pese a que siempre se ha mantenido constante la responsabilidad de cuidar a los clientes, en los últimos años ha experimentado grandes cambios, gracias, en parte, a las nuevas tecnologías, lo cual ha hecho que se pueda ejecutar de una manera más eficiente ese acercamiento y entendimiento del cliente (Carreño, 2017). En Colombia estas estrategias van tomando cada vez más fuerza; el papel de las redes sociales, como Twitter, Facebook e Instagram, ha adquirido gran importancia como canales de comunicación y comercialización masivos, dado que resultan mucho más baratas y prácticas, además de tener en cuenta su influencia e interacción en el mercado actual. Relacionado con el servicio al cliente, García (2016) apunta que este posee una serie 
de características que lo definen, dentro de las cuales están su intangibilidad, heterogeneidad, simultaneidad en el momento de la producción y el consumo, y la imposibilidad de ser almacenado, es decir, es perecedero.

De manera relacionada, hay que mencionar que la satisfacción plena del cliente es un elemento fundamental para que esa persona vuelva a consumir el servicio prestado; como señala Thompson (2005), con un excelente servicio se puede ganar un lugar en la mente del cliente, lo cual es un paso adelante para su satisfacción y lealtad; para ello, toda la organización debe actuar en función de ese objetivo. Así mismo, Jahanshahi et al. (2011) reconocen que existe una alta correlación entre los niveles del servicio al cliente y su satisfacción y lealtad. En cuanto a esto, Mendoza (2019) encontró que en el transporte aéreo las variables satisfacción del cliente y bajo costo no están relacionadas.

Unido a la satisfacción del cliente, otro factor fundamental es la calidad del servicio. Es una de las fuentes principales de valor para el cliente, el cual es cada vez más exigente en el consumo de los servicios que puedan satisfacer sus necesidades (Zárraga et al., 2018). Aunque no son lo mismo, la calidad del servicio es de alguna forma proporcional a la satisfacción; estudios como el de Carcausto (2016) indican una correlación positiva de 0.772 entre la calidad del servicio y la satisfacción del cliente. Las empresas deben procurar el suministro de un servicio con la mayor calidad posible, entendiendo que la calidad es un tema complejo y subjetivo, además de que ofrecer servicios con elevada calidad no es suficiente, pero sí necesario para lograr el éxito empresarial (Setó, 2004).

En cuanto al transporte aéreo, Huayanay y Romero (2018) reportaron que los equipos dentro de la aeronave, la estructura de los aviones y el servicio de comida y bebida a bordo son los elementos que más afectan la satisfacción del usuario. Pero en otro estudio llevado a cabo por Adeola y Adebiyi (2014) la satisfacción de los clientes se deriva principalmente de lo que ocurre en la primera etapa del servicio, es decir, de cómo las aerolíneas realizan sus procesos de emisión de boletos y reservas. También hay otras ideas, como la de Singh y Sirdeshmukh (2000), quienes proponen un marco para comprender la formación de la satisfacción, el cual relaciona la teoría de la agencia, que incorpora el precio o el número de competidores disponibles, con la teoría de la confianza, que tiene un acercamiento desde la psicología y toma en consideración elementos blandos del servicio. El estudio de Yu y Dean (2001) tiene un acercamiento similar, en el cual se incluyen tanto el componente cognitivo 
como el afectivo en la medición de la satisfacción, siendo el componente afectivo el mejor predictor de la lealtad.

Con respecto a la lealtad, Carvajal (2015) estableció que esta es consecuencia de la satisfacción, y que los elementos o subvariables que más tienen en cuenta los clientes son la atención, el producto, la marca, la innovación, el programa de fidelidad y el precio; así mismo, destaca que los programas de fidelidad son significativamente influenciadores del aumento en los valores de esta variable. Moliner y Berenguer (2011) confirman que el desempeño en el servicio contribuye positivamente en la satisfacción, al igual que el desempeño también tiene una influencia positiva en la conformación de la lealtad; y, de manera lógica, la satisfacción igualmente ejerce influencia positiva sobre la lealtad, aunque la relación entre satisfacción y lealtad es asimétrica, es decir, los consumidores leales normalmente están satisfechos, pero no todos los clientes satisfechos son leales (Oliver, 1999).

Ya en el campo del transporte aéreo, el estudio desarrollado por Pastrana-Martínez et al. (2020) concluye que "la lealtad se refleja en el gusto del cliente por volar en la aerolínea, en su orgullo de decir a otros que viaja en ella y en su percepción de que la empresa lo anima para que la utilice" (p. 492). Pero, dice también Oliver (1999) que la lealtad es una meta inalcanzable para algunas empresas, debido a la naturaleza del producto y al desinterés del consumidor, por lo que, para estas compañías, la satisfacción de sus clientes sigue siendo el objetivo por el que deberían esforzarse.

Los estudios clásicos indican que la lealtad es consecuencia directa de la satisfacción del cliente, aunque estudios recientes cuestionan este vínculo. Para Bravo et al. (2005), la lealtad de los pasajeros de las líneas aéreas está determinada por la satisfacción, la confianza y el compromiso. En contraste con lo anterior, Jiang y Zhang (2016) concluyeron que la calidad del servicio es una variable importante que influye en la satisfacción del cliente, pero no encontraron que el servicio satisfactorio se convirtiera en una mayor lealtad entre los viajeros de negocios. Por otro lado, una importante investigación de Cronin et al. (2000) evidenció empíricamente que, cuando se estudian en conjunto, variables como la satisfacción, la calidad del servicio y su valor influyen notablemente en el comportamiento del consumidor. 
Ciertamente, hay resultados contradictorios en cuanto a la relación de las variables antes mencionadas. Esto lo investigaron Szymanski y Henard (2001), quienes confirmaron que la equidad y la justicia en el servicio está relacionada con la satisfacción del cliente; también documentaron que los métodos que utilizan en cada investigación condicionan la fuerza con que se relacionan las variables satisfacción, calidad, lealtad, entre otras. Existe un importante número de variables o factores que influyen en la satisfacción del cliente. Otro ejemplo de ello lo mencionan Spreng et al. (1996) cuando proponen un modelo de formación de la satisfacción basado en la teoría de la desconfirmación de expectativas y que asume el impacto de la comunicación del mercadeo en la variación de la satisfacción.

Una de las formas de estructurar el servicio para poderlo evaluar es de acuerdo con el momento en que sucede; este se puede estructurar en pretransacción, transacción y postransacción (Ballou, 2014). De manera similar, Jochen (2015) indica que el proceso se divide en los momentos: previos a la adquisición, el consumo y la fase posterior al consumo. Entonces, el estudio de satisfacción y lealtad del pasajero debe abordar los tres momentos para que este sea lo más completo posible.

\subsection{Las aerolíneas}

Conforme con la Organización de Aviación Civil Internacional (OACI, 2015), las aerolíneas son empresas que se dedican al traslado de pasajeros o carga por avión. El sector del transporte aéreo es bastante complejo, pues existen organizaciones que transportan personas y carga de manera permanente, mientras que también hay otras compañías que trasladan a sus clientes de la forma acordada entre ellos. Estas últimas son llamadas charter.

Dentro de las aerolíneas de pasajeros, se han desarrollado dos modelos de negocios: las aerolíneas tradicionales o convencionales, que fueron las primeras en crearse desde los inicios de la aviación comercial; y más recientemente surgieron las aerolíneas de bajo costo. En 1949, la aerolínea Pacific Southwest Airlines empezó operaciones, siendo su principal estrategia de negocio los precios bajos. Luego en la década de los setenta del siglo xx, con la desregulación del transporte aéreo en Estados Unidos, otras aerolíneas comenzaron a practicar estas estrategias. Ya cerca al año 1980 se potencializa el modelo teniendo como una de sus protagonistas a Southwest Airlines. Luego estas prácticas fueron extendidas por Europa por el año 1990 cuando Ryanair adaptó ese modelo, y de allí a todo 
el mundo (Ginieis et al., 2017), aprovechando épocas de crecimiento económico. Hoy en día, una low cost se define como una aerolínea de precios bajos y servicios limitados, al compararse con las líneas aéreas convencionales (Canseco et al., 2015).

Ya en la primera década del siglo xxi varias líneas aéreas iniciaron operaciones en Australia y Asia. La secuencia de inicio de actividades en cada parte del mundo hace ver que el modelo de negocio que caracteriza a una low cost se desarrolla de mejor manera en los mercados liberalizados (Sheth et al., 2007).

\subsection{Aerolínea de bajo costo versus aerolínea convencional - servicios}

Según la Asociación Internacional de Transporte Aéreo (IATA, 2014), una operación low cost se define como aquella que incluye rutas cortas, operaciones punto a punto, uso de aeropuertos regionales o secundarios; además de eso, su target son los viajeros de ocio, sus aeronaves tienen una sola clase de servicio, generalmente no poseen programas de viajero frecuente, los servicios son bastante finitos, con cargos adicionales por algunos servicios, su principal estrategia son los precios bajos, su principal canal de comercialización son las plataformas virtuales y sus propias páginas web, sus aeronaves normalmente son de un solo modelo y se mantienen en constante operación, aparte de tener una estructura sencilla de administración.

Las aerolíneas de bajo costo no sirven alimentos, el pasajero tiene derecho a llevar solo un bolso de mano, todo debe ser pagado en efectivo, no posee cupones ni promociones para viajeros frecuentes, es decir, todo servicio extra al derecho de su asiento debe ser pagado (Rodríguez, 2018). Las aerolíneas regulares normalmente venden el boleto incluyendo varios o todos los servicios anteriormente mencionados, además de que sus aviones están configurados con varias clases de asientos, por lo que las aeronaves de las low cost poseen una mayor densidad de asientos, una mayor capacidad de pasajeros, debido al menor espacio para las piernas y menos reclinación de la clase económica.

Comenta Tipiani (2019) que las líneas aéreas tradicionales y low cost están acercando sus prácticas en cuanto a la selección de los asientos. Los clientes de las operadoras de bajo costo pueden reservar número de butaca solo en el proceso de facturación en línea 
y, además, deben pagar por el servicio. En cambio, las aerolíneas tradicionales usualmente permiten la escogencia de silla de manera gratuita cuando se hace la reserva.

En cuanto a canales de venta, las aerolíneas de bajo costo privilegian la comercialización de sus boletos a través de internet, preferiblemente por medio de su propia plataforma, con lo cual pagan menos comisiones a los intermediarios. Por su parte, las aerolíneas convencionales poseen mayores opciones para la comercialización (Rodríguez, 2018). Otro elemento importante del servicio aéreo es el equipaje; en este caso, las aerolíneas de bajo costo no incluyen el equipaje dentro del precio del boleto, sino que se cobra por aparte, lo que lo hace más costoso en muchas ocasiones, al compararse con las tradicionales.

En la operatividad, cuando así lo permite la infraestructura, las aerolíneas de bajo costo utilizan aeropuertos pequeños, normalmente ubicados más lejos de la ciudad, que tienen tasas aeroportuarias más bajas comparados con los aeropuertos principales. Estos aeropuertos secundarios tienen mayor disponibilidad de slots, por lo que los vuelos tienen una tasa de cumplimiento de sus itinerarios superior, se retrasan menos (Canseco et al., 2015). Las aerolíneas convencionales siguen usando los aeropuertos principales, los cuales son más cómodos para los pasajeros, pero requieren un pago mayor de tasas aeroportuarias.

Otra diferencia relevante entre aerolíneas convencionales y de bajo costo está relacionada con las rutas que operan, específicamente con la duración de estas. Las líneas aéreas de bajo costo se concentran en rutas cortas, ya que estas ofrecen mayores eficiencias y, por lo tanto, mejoras en los márgenes de utilidades, mientras que las tradicionales, en su propósito de prestar el mejor servicio posible, incluyen rutas largas y con escalas.

Por otra parte, de acuerdo con Casagualda y García (2013), las aerolíneas convencionales poseen varios tipos de aeronaves dentro de su flota, porque tienen una diversidad de rutas que cubrir, mientras las low cost emplean un solo tipo de aeronave, lo que les permite tener una operatividad, mantenimiento y administración más sencillos.

Las aerolíneas convencionales y de bajo costo han ido convergiendo en precios; las tradicionales están disminuyendo costos, emitiendo promociones y brindando diversas opciones a sus clientes sobre las tarifas disponibles como una de sus estrategias de competencia con 
las low cost. Por su parte, las líneas de bajo costo, para atraer a viajeros de negocios, han propuesto algunos servicios adicionales (Gherraby, 2016).

En el mercado colombiano, la aerolínea de bajo costo EasyFly fue la primera en comenzar a operar en 2007, haciéndolo en rutas regionales (Parra \& Mateus, 2020). Luego se incorpora Viva Colombia, operando vuelos internacionales con destino a Lima y Panamá; viendo los buenos resultados de estas dos aerolíneas, otros inversionistas crearon la aerolínea Wingo, de capital colombo-panameño (Condo et al., 2018).

\section{Elementos metodológicos}

Las estrategias metodológicas dan cuenta de los procesos y formas que se siguieron en el desarrollo de la investigación. Este proyecto tuvo un enfoque cuantitativo, que, según Hernández y Mendoza (2018), está relacionado con conteos numéricos y métodos matemáticos, pero además representa un conjunto de procesos organizados de manera secuencial, partiendo de una idea que se delimita y de donde surgen preguntas, a las cuales se les da respuesta haciendo recolección de información, análisis estadísticos y concluyendo sobre lo observado. Adicionalmente, fue una investigación de corte transversal, no experimental, teniendo como sujetos de estudio a los pasajeros de las aerolíneas que operan en el mercado doméstico colombiano.

Con respecto al alcance de la investigación, esta se diseñó para ser descriptiva, aunque se llegaron a establecer correlaciones entre las variables consideradas. Los estudios con alcance descriptivo "pretenden medir o recoger información de manera independiente o conjunta sobre los conceptos o las variables a las que se refieren" (Hernández et al., 2014, p. 92). Por otra parte, las investigaciones con alcance correlacional procuran conocer la asociación que puede existir entre dos o más variables, en este caso la satisfacción y la lealtad.

Para dar cumplimiento a los objetivos de la investigación, se consultó a los clientes de las aerolíneas de bajo costo y a los de las aerolíneas convencionales que operan en el país; desde las instalaciones del Aeropuerto Internacional El Dorado de Bogotá, se evaluaron 
las experiencias del servicio que recibieron los pasajeros que volaron en alguna de estas aerolíneas a nivel nacional, aplicando un instrumento tipo encuesta durante los meses de abril y octubre de 2019, el cual, conforme con Medina et al. (2012), es el instrumento por predilección de la investigación cuantitativa; la recolección de datos se llevó a cabo a través de fuentes primarias, es decir, encuestando a los individuos integrantes de la población en estudio (Torres et al., 2019).

La encuesta fue diseñada de acuerdo con las variables en estudio: satisfacción y lealtad; el instrumento constó de 21 preguntas y abarcó todo el proceso que desarrolla el cliente con la aerolínea, desde la compra del boleto hasta la salida en el aeropuerto de destino. Las interrogantes en las cuales se indagaba sobre la satisfacción en cada uno de los momentos del servicio tuvieron como posibles respuestas una escala ordinal de cinco valores, al estilo de Likert, muy utilizada en los estudios de opinión de usuarios de servicios (Llanos Zavalaga et al., 2001), donde 1 correspondía a 'totalmente insatisfecho'; 2, a 'insatisfecho'; 3, a 'neutral'; 4, a 'satisfecho', y 5, a 'totalmente satisfecho'. De modo similar, para evaluar las futuras repeticiones del servicio, relacionadas con los niveles de lealtad, se consideró la escala con valores de 1 a 5, donde 1 era 'nunca más'; 2, 'posiblemente no'; 3, 'no lo sé'; 4, 'posiblemente sí'; y 5, 'seguramente sí'. Para los diversos análisis se agruparon los valores 1 y 2 como evaluación negativa (insatisfacción o falta de lealtad), y los valores 4 y 5 como evaluación positiva (satisfacción y lealtad). La validez de contenido de la encuesta se logró gracias a la revisión y retroalimentación de dos docentes del área de servicio al cliente, con estudios posgraduales relacionados, y dos gerentes de aerolíneas colombianas, relacionados también con el servicio al cliente.

Se tomó como población a los pasajeros que abordaron algún vuelo nacional con destino al aeropuerto El Dorado en la ciudad de Bogotá durante el año 2019. De acuerdo con las estadísticas presentadas por la Aerocivil (2019), hubo un total de 3781495 pasajeros, lo cual se puede considerar una población infinita. Según lo propuesto por Aguilar (2005), para un $95 \%$ de nivel de confianza y un $5 \%$ de error máximo, la muestra mínima era de 384 elementos; se aplicaron 443 encuestas, pero se descartaron 22 por no cumplir con todos los parámetros establecidos, procesándose finalmente un total de 421 encuestas válidas.

Cada una de las respuestas de los encuestados era vaciada en la aplicación Formulario de Google, de la cual se descargaron, en archivo tipo xlsx, todos los datos recolectados. 
Luego, estos datos recolectados se procesaron en hojas de cálculo, mediante el software Excel, haciendo una revisión sistemática de la información.

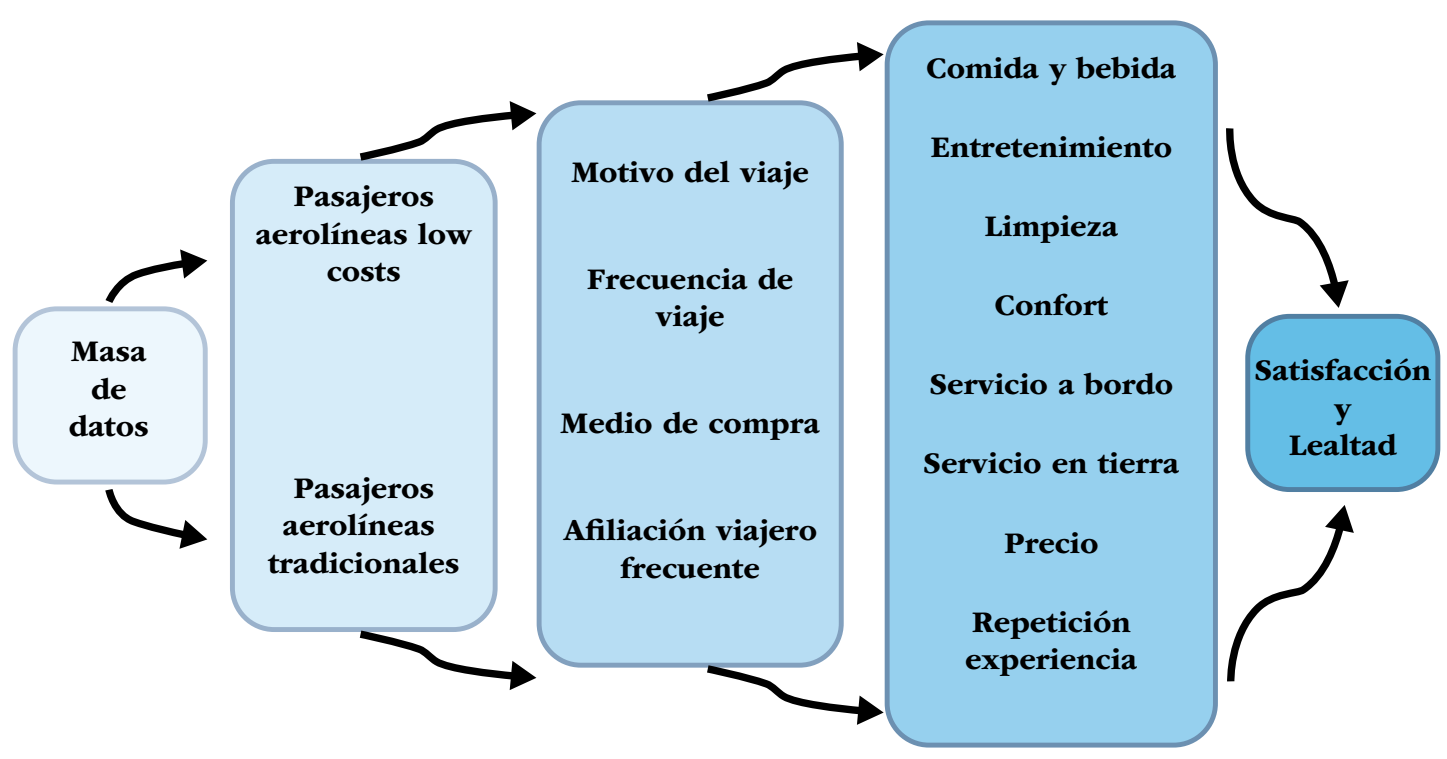

Figura 1. Proceso de transformación de los datos

Fuente: elaboración de los autores.

Como se observa en la figura 1, la masa de datos se organizó por el tipo de aerolínea donde colaron los pasajeros (low cost y tradicionales). A su vez se aplicaron distintos filtros de acuerdo con algunas características del pasajero (motivo de viaje, frecuencia de viaje, medio de compra, afiliación viajero frecuente), para poder evaluar los distintos elementos del servicio (comida y bebida, entretenimiento, limpieza, confort, servicio a bordo, puntualidad, servicio en tierra, precio y repetición de la experiencia), y, finalmente, poder relacionar el comportamiento de las variables principales (satisfacción y lealtad).

\section{Resultados y discusión}

A continuación, se analizan los resultados más significativos, después de procesar 421 encuestas aplicadas a los viajeros del aeropuerto El Dorado, en Bogotá, y discriminando estos resultados entre las aerolíneas de bajo costo y las tradicionales. El primer resultado 
interesante, observado en la figura 2 , fue el motivo de viaje por el que cada persona ocupó el vuelo; las opciones de turismo, compromisos familiares y estudio son las más reportadas por los pasajeros de las aerolíneas de bajo costo, mientras que los viajes por motivos laborales son más reportados en las líneas aéreas tradicionales.

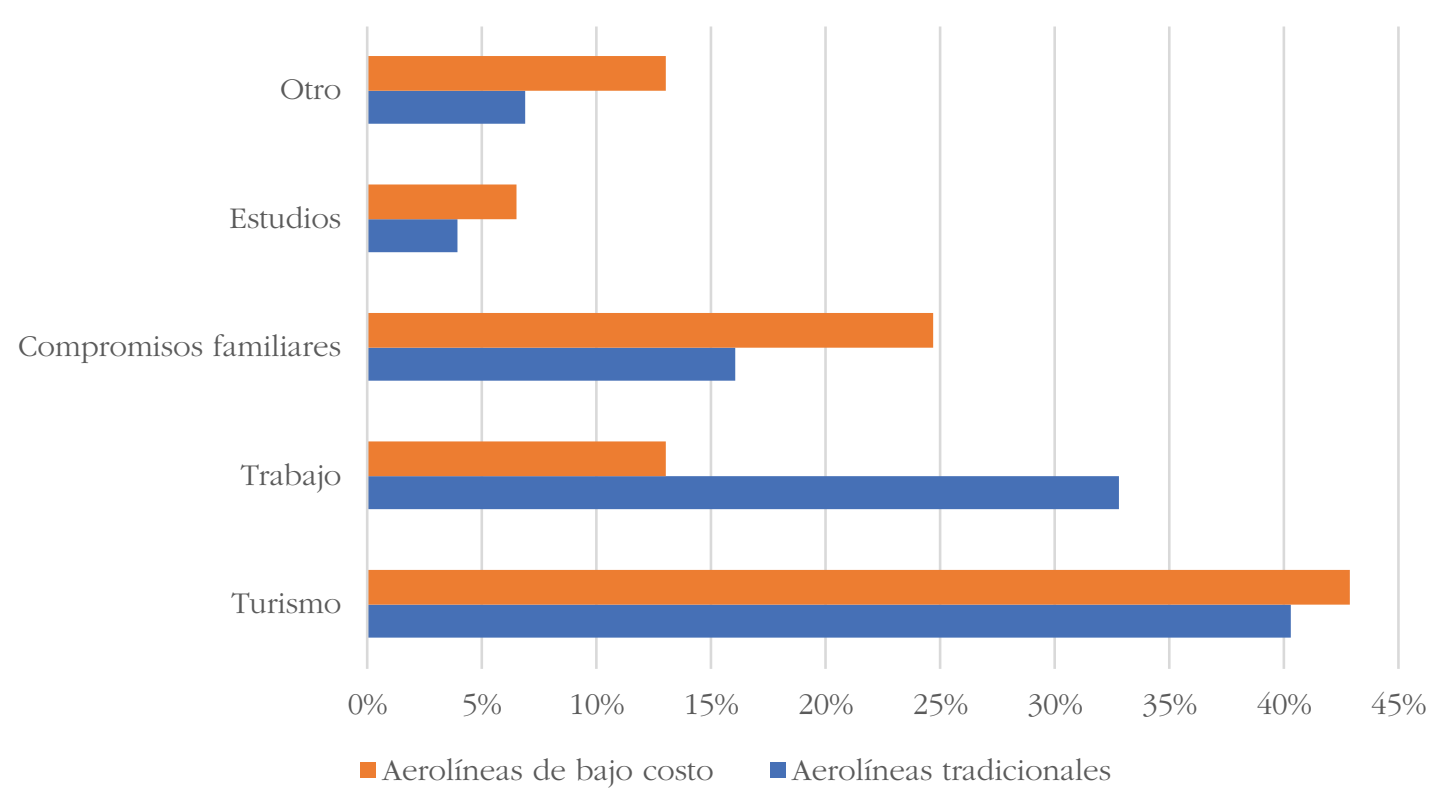

Figura 2. Motivo del viaje

Fuente: elaboración de los autores.

Pareciera ser que los viajes por estudio, compromisos familiares y turismo incluyen a un sector del mercado que busca más la economía, esto comparado con los viajes por motivos de negocios o laborales, cuyos pasajeros anteponen los servicios adicionales, tales como derechos preferenciales en las colas, salas VIP, opciones para cambio de pasajes, a la economía que podrían tener otras opciones de viaje. Además, hay un factor adicional: los boletos para vuelos con motivos laborales normalmente son adquiridos por algún departamento de la empresa donde labora el pasajero, quienes poseen políticas estandarizadas para la adquisición de estos servicios, que privilegian la compra de tarifas completas, como las que ofrecen las aerolíneas tradicionales. Eso concuerda con los resultados de Luchessi et al. (2015): los clientes que viajan por motivos de turismo normalmente pagan su propio boleto, por lo tanto, buscan alguna economía en la operación de compra, mientras que para los que viajan por negocios sus boletos son comprados por algún funcionario de la organización donde trabajan, y se anteponen otros elementos como la hora del vuelo antes que el precio. 
Luego se observan otros datos en la figura 3, relacionados con la frecuencia de viaje de cada persona encuestada. Aquí se encuentra que las frecuencias más altas, es decir, una vez al mes o más, y menor de una vez al mes y mayor de una vez al año, corresponden a la mayoría de los viajeros en aerolíneas tradicionales, mientras las más bajas, una vez al año y menos de una vez al año, involucran en mayor medida a los pasajeros de las low cost. Este resultado coincide o está relacionado con el anterior, ya que los pasajeros de trabajo o negocios tienen una mayor frecuencia de viaje, y ellos también ocupan el servicio de las líneas tradicionales.

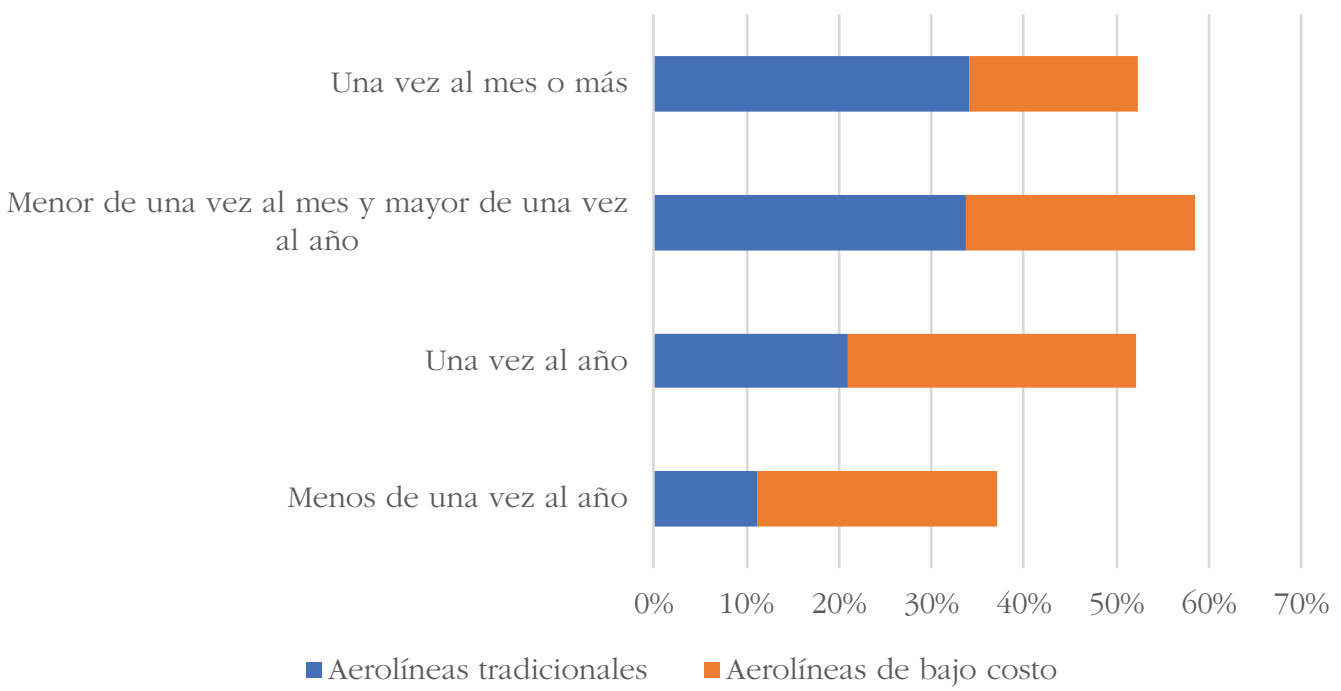

Figura 3. Frecuencia de viaje

Fuente: elaboración de los autores.

Es importante tomar en cuenta este resultado, pues los viajeros que utilizan una vez al mes o más el servicio, es decir, los pasajeros de mayor frecuencia, son los clientes sobre los cuales es necesario implementar las campañas de inscripción a los programas de fidelización que cada una de las aerolíneas posea (Camacho, 2013). Las aerolíneas tradicionales, al tener un mayor porcentaje de clientes con alta frecuencia de compra, entonces tienen mayor posibilidad de conseguir la lealtad de estos, incluso se podría asumir que ya poseen mayor porcentaje de fidelización que las compañías de bajo costo, según este resultado.

En cuanto al medio por el cual se realizó la compra, el resultado observado en la figura 4 indica una alta similitud entre los dos tipos de aerolíneas. El medio más ampliamente usado para la compra de los boletos aéreos, tanto para las aerolíneas de bajo costo como para las 
tradicionales, es el internet, sea a través de alguna plataforma especializada en la venta de boletos o directamente por la página web de la aerolínea.

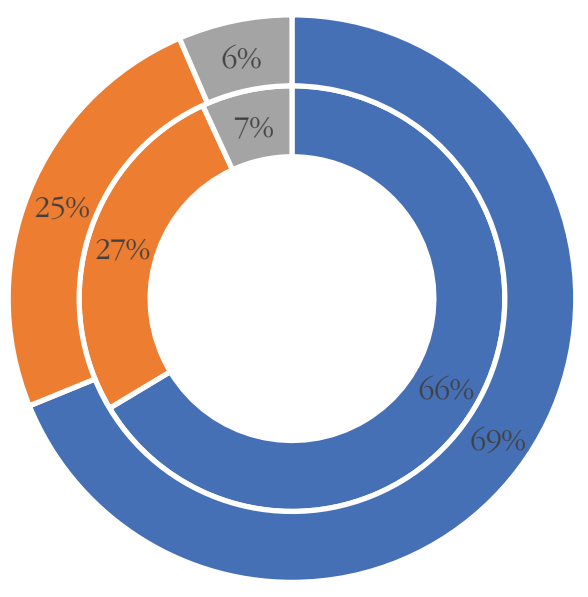

Figura 4. Medio de compra
- Compra online

- Agencia de Viajes

- Oficina de la aerolínea

Fuente: elaboración de los autores.

Como lo comentan Garmendia y Cubides (2020), la comercialización de boletos aéreos a través de internet ha tenido un crecimiento significativo en los últimos años; hay que recordar que al inicio del comercio electrónico las aerolíneas de bajo costo fueron las primeras en usar este canal de comercialización, con la intención de reducir costos en ese proceso; pero luego, a medida que este medio de compra se fue popularizando, las aerolíneas tradicionales observaron la necesidad de incursionar en él, por lo que actualmente solo se evidencia una diferencia del 3\% entre ambas; las aerolíneas de bajo costo en Colombia venden un $69 \%$ de sus pasajes a través de internet y las tradicionales, un $66 \%$.

También es muy importante evaluar distintos elementos, tanto servicios particulares como aspectos relevantes del servicio en general. En la figura 5 se pueden apreciar estos resultados; con respecto a la comida y bebida, entretenimiento y confort, las aerolíneas tradicionales obtienen unos resultados de satisfacción mucho más altos que las de bajo costo, observándose una diferencia de por lo menos un $24 \%$. Estos rubros son precisamente los que las aerolíneas de bajo costo han eliminado de sus servicios: las comidas y bebidas se compran en el vuelo, no poseen pantallas de Tv en cada uno de sus asientos y sus aeronaves 
poseen una sola clase, hay un mayor número de pasajeros por metro cuadrado disponible dentro de la cabina de pasajeros (Javurek, 2013).

En este último aspecto también influye el tipo de aeronave que se utiliza: para estos vuelos domésticos, las aerolíneas tradicionales utilizan aviones como la familia de los Airbus A320, mientras que las de bajo costo usan aeronaves turbohélice como los ATR 42 y 72. Estos últimos tienen una cabina de pasajeros más angosta, lo cual da la sensación al pasajero de menos espacio disponible. Respecto a lo anterior, Forgas et al. (2011) encontraron que "la percepción de las condiciones en las aeronaves, como la comodidad y el espacio entre asientos, juegan en el modelo conceptual planteado una relación negativa de lealtad en las compañías de bajo coste" (p. 9).

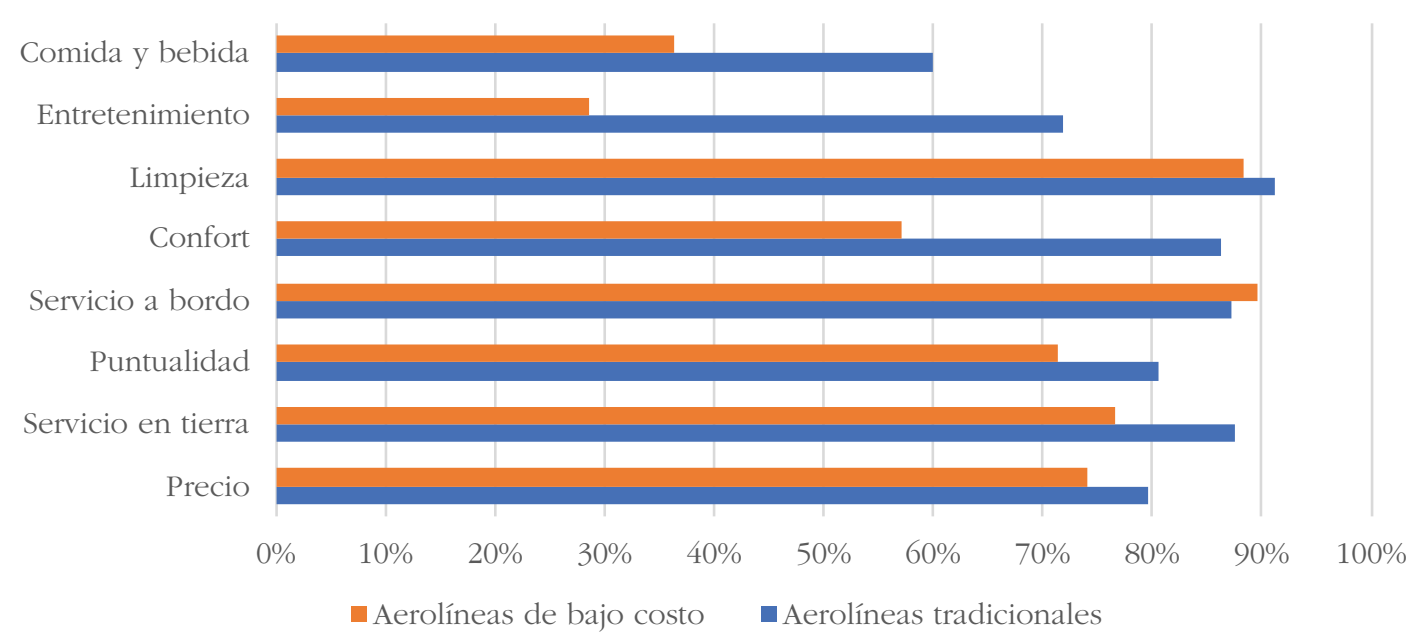

Figura 5. Satisfacción de diversos elementos del servicio

Fuente: elaboración de los autores.

Hay otras categorías como la limpieza, la puntualidad y el servicio en tierra, que aparecen en la mayoría de los modelos descritos por Ibarra y Paredes (2016), en los que la diferencia de satisfacción es menor al $10 \%$, pero siempre a favor de las aerolíneas tradicionales; aunque la diferencia es menor, siguen prevaleciendo los servicios prestados por las aerolíneas tradicionales en estos rubros. La única categoría en que los pasajeros de las aerolíneas de bajo costo reportan una mayor satisfacción sobre los usuarios de las líneas aéreas tradicionales es en el servicio a bordo, lo cual resulta importante, pese a que esta ventaja sea solo del $3 \%$. Con este resultado se puede percibir la claridad del cliente para evaluar el servicio, ya que, aunque no están satisfechos con la comida y bebida, ni el entretenimiento o el confort 
en las low cost, igualmente aprecian en buena manera la atención que el personal de la aerolínea les brinda en la aeronave, logrando un $90 \%$ de satisfacción.

Luego, en la figura 6 se perciben resultados más integrales y concluyentes. Cuando se les preguntó a los usuarios sobre la recompra del servicio en la aerolínea por la que viajaron, un $64 \%$ de los pasajeros de las compañías de bajo coste aseguraron que seguirían viajando por esa aerolínea, mientras un $89 \%$ de los viajeros en las aerolíneas tradicionales confirmaron que preferirían seguir viajando en esta.

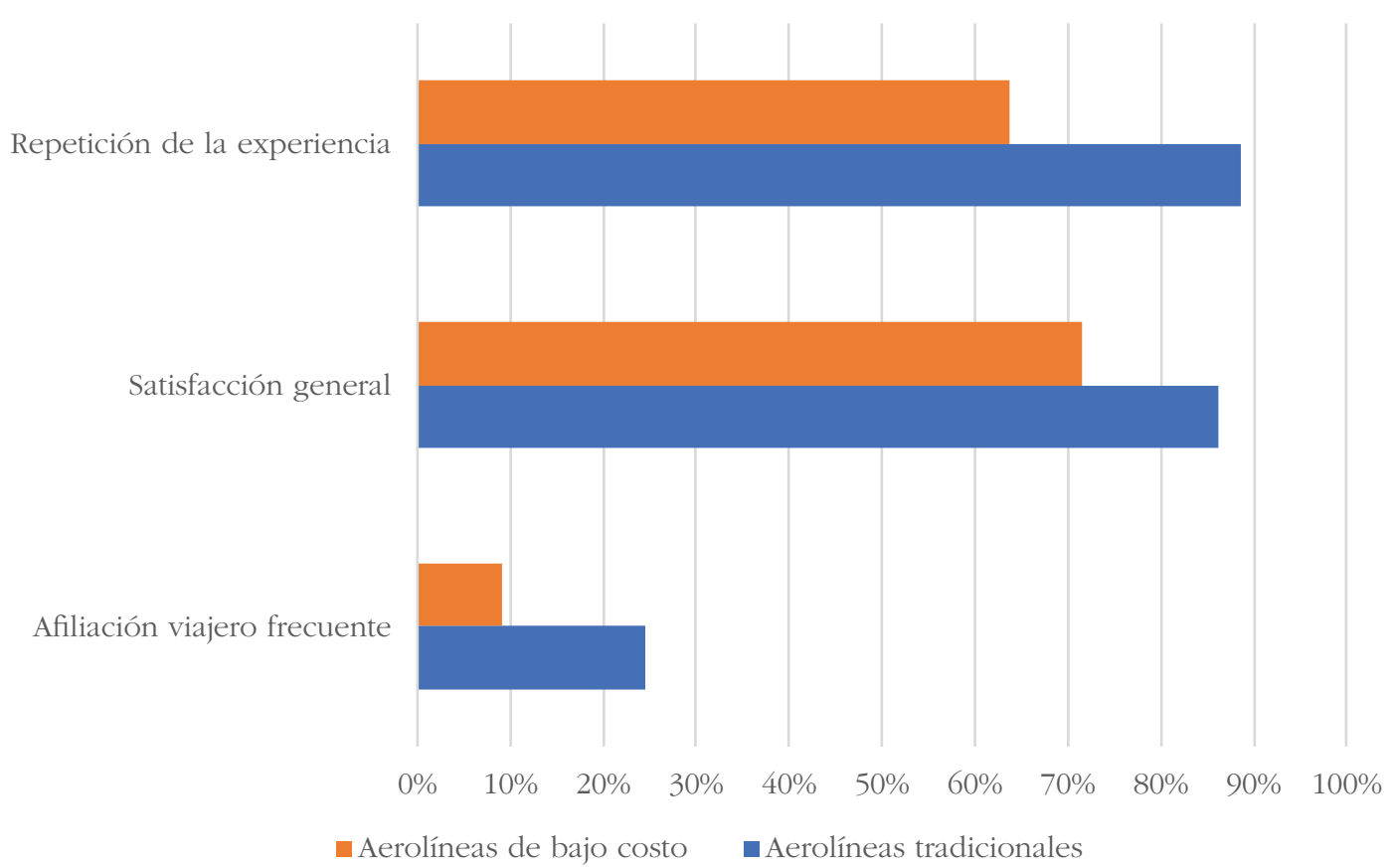

Figura 6. Satisfacción y lealtad

Fuente: elaboración de los autores.

En cuanto a la satisfacción general por toda la experiencia, los usuarios de las aerolíneas de bajo costo reportan un $71 \%$, mientras las tradicionales obtuvieron un $86 \%$ de satisfacción por parte de sus viajeros. Estos resultados que reflejan la opinión general de los pasajeros, en cuanto a su satisfacción y lealtad con la aerolínea, están relacionados con el número de clientes afiliados a un programa de viajero frecuente; precisamente el objetivo de estos programas es contribuir a la recompra y la lealtad del cliente. Entonces se observa que solo un $9 \%$ de los pasajeros de las low cost están afiliados con esas aerolíneas, mientras que un $25 \%$ de los clientes de las tradicionales tienen afiliación de viajero frecuente con estas. En las aerolíneas 
tradicionales se cumple en mejor medida un círculo virtuoso, en donde la satisfacción del cliente hace que quiera volver a tener la experiencia del vuelo con esta, además de que los beneficios de los programas de viajero frecuente hacen más atractiva esta opción, lo cual es confirmado por Contreras (2016).

En el estudio presentado por Maynez et al. (2020) sobre el transporte aéreo mexicano, encontraron que "ni en términos actitudinales ni comportamentales, existen discrepancias [...] ambos modelos de negocio, con ofertas de valor distintas, logran que sus clientes sean leales y que tengan la intención de seguir consumiendo" (p. 18). Este resultado contrasta con lo observado en el mercado colombiano, donde sí se evidencian diferencias sustanciales entre la satisfacción y la lealtad de los pasajeros low cost, comparados con los viajeros de las aerolíneas tradicionales, lo cual confirma los resultados de Cronin et al. (2000) al observarse la proporcionalidad entre la satisfacción y la repetición de la experiencia: a mayor satisfacción, mayor intención de repetir.

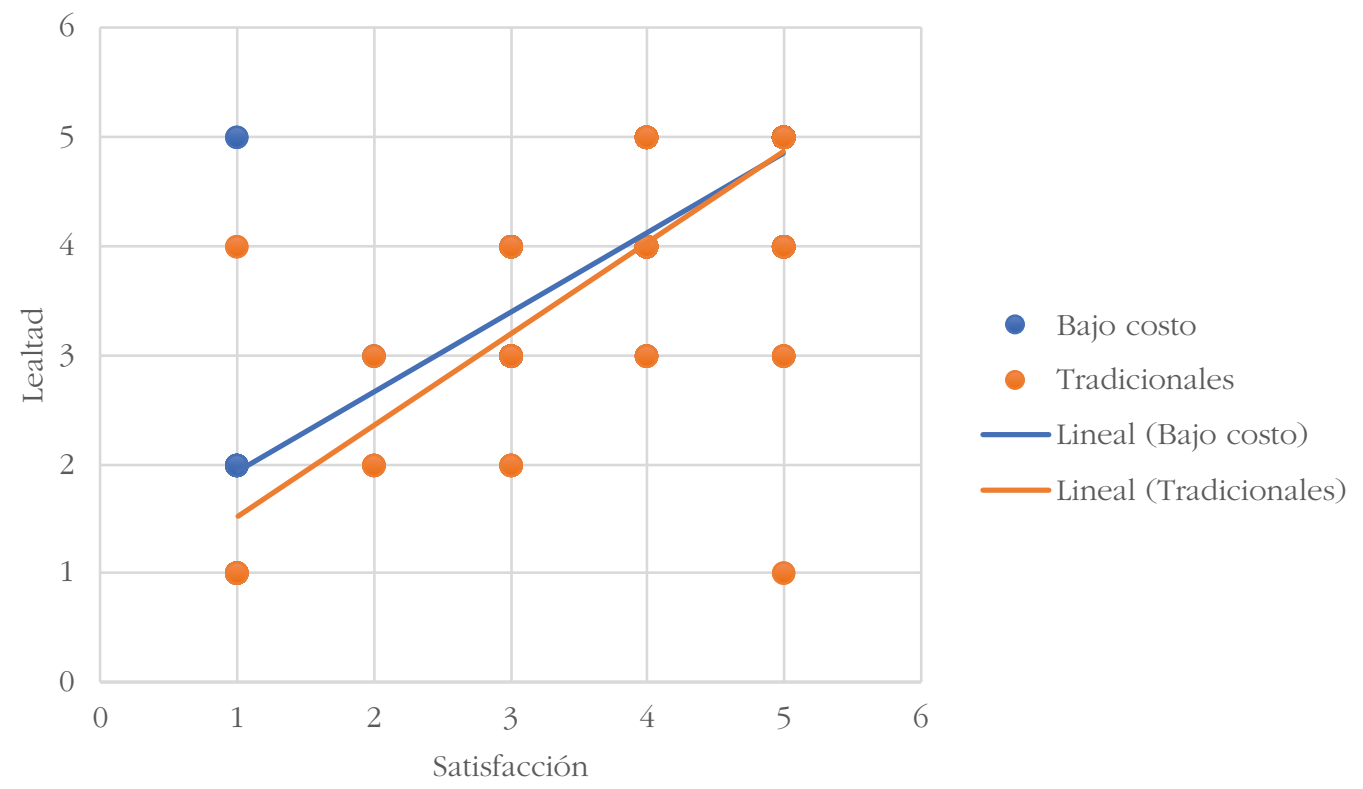

Figura 7. Dispersión y tendencia de satisfacción y lealtad

Fuente: elaboración de los autores.

Finalmente, en la figura 7 se presenta la dispersión y la tendencia de las variables lealtad y satisfacción. Efectivamente, se observa una correlación positiva entre estas dos variables tanto para las aerolíneas de bajo costo como para las tradicionales. Para las aerolíneas 
tradicionales, se obtuvo un coeficiente de correlación de 0.81; y, para las compañías de bajo costo, el coeficiente calculado fue de 0.85. En ambos casos se obtuvieron valores que, al decir de Benesty et al. (2009), representan una asociación alta entre ambas variables. Estos resultados tienen afinidad con los hallados por Ibarra y Paredes (2016), al expresar que la lealtad del pasajero de las aerolíneas mexicanas es explicada en un $72.3 \%$ por la satisfacción.

\section{Conclusiones}

El surco que separa a las aerolíneas convencionales de las de bajo costo está cada vez más difuminado. Esto se observa, por ejemplo, en los canales de comercialización: hace unos años atrás, la venta de boletos a través de canales virtuales era una actividad exclusiva de las empresas de transporte aéreo que querían reducir costos, entonces las aerolíneas tradicionales lo consideraban un mal servicio, con algo de razón, porque no era una actividad tan amigable para el usuario. Hoy en día, gracias a los avances tecnológicos, el mercadeo por internet es una actividad muy confiable y sencilla para el usuario, por lo que lo utilizan masivamente todas las aerolíneas, tanto convencionales como low cost.

Estos avances tecnológicos también deben servir para otorgar un servicio más personalizado, tomando en consideración las características de cada pasajero, como, el género, ya que se han determinado comportamientos muy distintos entre hombres y mujeres. Igualmente, se constató que cada modelo de aerolíneas tiene su nicho de mercado: las aerolíneas tradicionales son las preferidas de los viajeros de negocios, mientras que las low cost son las favoritas de los turistas y los viajeros por compromisos familiares.

Aunque las diferencias entre las low cost y las aerolíneas tradicionales se han estrechado, todavía hay elementos del servicio en los que se observan en mayor medida, tales como los servicios de alimentos y bebidas, entretenimiento y el confort dentro de la aeronave. Efectivamente, los dos primeros no son una opción en las low cost colombianas, mientras que respecto al confort es también notoria la disminución de espacio entre asientos en los aviones de las aerolíneas de bajo costo. 
Las aerolíneas de bajo costo son una opción para muchos ciudadanos que necesitan o desean realizar un viaje, sea por negocios, turismo, estudio o compromisos familiares, pero así mismo es cierto que todavía existe diferencia en cuanto a la satisfacción que producen en sus pasajeros, comparada con las aerolíneas convencionales. Parte de este resultado es consecuencia de la falta de información que tiene el pasajero en cuanto a sus derechos al comprar un boleto en una low cost. Por esto, las aerolíneas de bajo costo deben mejorar sus estrategias de comunicación para que los clientes tengan mayor certeza de los servicios que están comprando y que, al iniciar la experiencia del viaje, sus expectativas estén más alineadas con la realidad, para que luego la satisfacción y la lealtad se incrementen como consecuencia del cumplimiento de las expectativas.

Tanto en las aerolíneas tradicionales como en las low cost la satisfacción es un buen predictor de la lealtad. Aunque existen algunas investigaciones que no confirman esta relación, en el caso del transporte aéreo sí se puede observar ese orden lógico, con lo cual también se puede expresar que los usuarios del transporte aéreo son consumidores inteligentes que aprecian la calidad del servicio y actúan en consecuencia.

La satisfacción de los clientes en el transporte aéreo es proporcional a su lealtad. El modelo de negocio de bajo costo hace menos probable que el consumidor esté satisfecho y, por lo tanto, es más difícil que sea leal a la marca, esto en el contexto colombiano, donde el mercado está todavía entendiendo y adaptándose al servicio low cost. Los pasajeros de las aerolíneas tradicionales tienen un mayor grado de satisfacción, así también demuestran mayor lealtad a la empresa. Ello también está relacionado con los programas de viajero frecuente, los cuales parecieran estar logrando su objetivo: la recompra del servicio. Entonces, es importante que las aerolíneas tradicionales sigan ofreciendo ese beneficio adicional con el cual logran un poco más de diferenciación con las low cost.

Que hayan pocas marcas en los mercados de transporte aéreo ayuda a que los pasajeros no tengan muchos elementos de cotejo, por lo tanto, la lealtad es un poco más fácil de lograr si se compara con otros mercados de servicios donde hay mayor competencia, es decir, donde existe más diversidad de marcas. 


\section{Referencias}

Adeola, M. M., \& Adebiyi, S. O. (2014). Service quality, perceived value and customer satisfaction as determinant of airline choice in Nigeria. International Letters of Social and Humanistic Sciences, 20(1), 66-80. Available from https://www.ceeol.com/search/viewpdf?id=579107

Aerocivil. (2019). Estadísticas operacionales —transporte aéreo origen destino-estadísticas de origen destino de diciembre 2019. Recuperado de http://www.aerocivil.gov.co/atencion/ estadisticas-de-las-actividades-aeronauticas/boletines-operacionales

Aguilar, S. (2005). Fórmulas para el cálculo de la muestra en investigaciones de salud. Revista Salud en Tabasco, 11(1-2), 333-338. Recuperado de https://www.redalyc.org/ pdf/487/48711206.pdf

Arif, M., Gupta, A., \& Williams, A. (2013). Customer service in the aviation industry. An exploratory analysis of UAE airports. Journal of Air Transport Management, 32, 1-7. https:// doi.org/10.1016/j.jairtraman.2013.05.001

Ballou, R. (2014). Logística, administración de la cadena de suministros ( $6^{\mathrm{a}}$ ed.). México: Pearson Educación.

Barría, C. (2020). Coronavirus: el impacto económico "sin precedentes" del Covid-19 en las aerolineas (y cómo puede afectar el precio de los pasajes). вBC News. Recuperado de https:// www.bbc.com/mundo/noticias-51757957

Benesty, J., Chen, J., Huang, Y., \& Cohen, I. (2009). Pearson correlation coefficient. In Noise reduction in speech processing. Berlin-Heidelberg: Springer.

Bravo, M., Vásquez, A., \& Zamora, J. (2005). Lealtad en el aire: factores reales y ficticios de la formación de la lealtad del pasajero de líneas aéreas. Revista Estudios y Perspectivas en Turismo, 14(2), 101-121. Recuperado de https://www.redalyc.org/pdf/1807/180713886001. pdf

Camacho, E. (2013). Alineación al éxito del negocio, el caso de las aerolíneas comerciales mexicanas (Tesis de maestría, Universidad Iberoamericana, Ciudad de México, México). Recuperado de http://ri.ibero.mx/bitstream/handle/ibero/1158/015738s.pdf?sequence=1

Canseco, A., Zúñiga, C., \& Blanco, L. (2015). Análisis estratégico sobre el desarrollo de las líneas aéreas de bajo costo en México. Revista Novoa Scientia, 7(15), 343-363. Recuperado de http://www.scielo.org.mx/scielo.php?pid=S2007-07052015000300343\&script=sci_arttext

Carcausto, Z. (2016). Relación entre calidad de servicio y satisfacción del cliente en el hotel Zakura de la ciudad de Juliaca (Trabajo de grado, Universidad Peruana Unión, Juliaca, Perú). Recuperado de https://repositorio.upeu.edu.pe/handle/upeu/879 
Carreño, C. (2017). La historia del servicio de atención al cliente en 500 palabras. Recuperado de https://www.brandembassy.com/es/resources/blog/la-historia-delservicio-de-atenci\%C3\%B3n-al-cliente

Carvajal, E. (2015). La fidelidad del consumidor en la distribución detallista (Tesis doctoral, Universidad Complutense de Madrid, España). Recuperado de https://eprints.ucm. es/32762/1/T36259.pdf

Casagualda, M., \& García, A. (2013). ¿Son las low-cost menos seguras que las aerolineas tradicionales? (Tesis de pregrado, Universidad Autónoma de Barcelona, España). Recuperado de https://ddd.uab.cat/record/113514

Condo, H., Fiorella, K., Pereyra, V., \& Grace, K. (2018). Estrategias de entrada y crecimiento utilizadas por las aerolíneas en su proceso de internacionalización al Perú (Tesis de maestría, Universidad Peruana de Ciencias Aplicadas, Lima, Perú). Recuperado de http:// hdl.handle.net/10757/623539

Contreras Serrano, C. G. (2016). Fidelización y rentabilización de usuarios de seguros todo riesgo de vehículos por medio de la venta cruzada y la venta escalonada. Un enfoque promocional para la industria aseguradora. Universidad E Empresa, 18(30), 143-157. https:// dx.doi.org/10.12804/rev.univ.empresa.30.2016.07

Cronin Jr., J. J., Brady, M. K., \& Hult, G. T. M. (2000). Assessing the effects of quality, value, and customer satisfaction on consumer behavioral intentions in service environments. Journal of retailing, 76(2), 193-218.

Forgas, S., Moliner, M., Sánchez, J., \& Palau, R. (2011). La formación de lealtad de un cliente de una compañía aérea: diferencias entre aerolíneas tradicionales y de bajo coste. Cuadernos de Economía y Dirección de la Empresa, 14(3), 162-172. Recuperado de https://www. sciencedirect.com/science/article/pii/S1138575811000119

García, A. (2016). Cultura de servicio en la optimización del servicio al cliente. Telos, 18(3), 381-398. Recuperado de https://www.redalyc.org/pdf/993/99346931003.pdf

Garmendia, J. (2019). Satisfacción y lealtad del cliente en las operaciones domésticas de las aerolíneas colombianas. Revista Fórum Empresarial, 24(1), 97-127. Recuperado de https:// revistas.upr.edu/images/forume/2019/v24n1/a4.pdf

Garmendia, J., \& Cubides, A. (2020). El servicio de plataformas en línea especializadas en venta de boletos aéreos. Suma de Negocios, 11(25), 116-124. https://dx.doi.org/10.14349/ sumneg/2020.v11.n25.a3

Gherraby, H. (2016). Análisis comparativo de la estructura de costes entre aerolineas tradicionales y de bajo coste: propuesta de mejora (Trabajo de pregrado, Universidad Politécnica de Valencia, Valencia, España). Recuperado de http://hdl.handle.net/10251/71337 
Ginieis, M., Sánchez, M., \& Campas, F. (2017). ¿Cuánto cuesta el personal según el tipo de aerolínea y su ubicación geográfica en Europa? Un análisis comparativo. Innovar, 27(65), 123-150. https://doi.org/10.15446/innovar.v27n65.65067

Hernández, R., Fernández, C., \& Baptista, P. (2014). Metodología de la investigación (6ª ed.). México: McGraw-Hill.

Hernández, R., \& Mendoza, C. (2018). Metodología de la investigación: las rutas cuantitativa, cualitativa y mixta. Ciudad de México: McGraw-Hill Interamericana Editores.

Huayanay, A., \& Romero, W. (2018). Estudio de la satisfacción del pasajero peruano de vuelos domésticos en base a la calidad de servicio de la aerolínea LC Perú (Trabajo de pregrado, Pontificia Universidad Católica del Perú, Lima, Perú). Recuperado de http://repositorio. pucp.edu.pe/index/handle/123456789/151697

IATA. (2014). Airline operational cost management guidance material manual.

Ibarra, L., \& Paredes, D. (2016). La calidad en el servicio y su efecto en la lealtad y satisfacción del pasajero en las aerolíneas de bajo costo. Sonora, México. Ágora de Heterodoxias, 2(1), 38-63. Recuperado de https://revistas.uclave.org/index.php/agora/article/view/223

Jahanshahi, A. A., Gashti, M. A. H., Mirdamadi, S. A., Nawaser, K., \& Khaksar, S. M. S. (2011). Study the effects of customer service and product quality on customer satisfaction and loyalty. International Journal of Humanities and Social Science, 1(7), 253-260.

Javurek, G. (2013). Empresas aéreas tradicionales y "low cost": entre el lucro, la seguridad y prácticas desleales. Revista Estudios de Derecho Empresario, 1, 238-242.

Jiang, H., \& Zhang, Y. (2016). An investigation of service quality, customer satisfaction and loyalty in China's airline market.Journal of Air Transport Management, 57, 80-88. https:// doi-org.loginbiblio.poligran.edu.co/10.1016/j.jairtraman.2016.07.008

Jochen, C. (2015). Marketing de servicios ( $7^{\mathrm{a}}$ ed.). Naucalpan de Juárez: Pearson Educación.

Llanos Zavalaga, F., Rosas Aguirre, A., Mendoza Requena, D., \& Contreras Ríos, C. (2001). Comparación de las escalas de Likert y vigesimal para la evaluación de satisfacción de atención en un hospital del Perú. Revista Médica Herediana, 12(2), 52-57.

Luchessi, S., Nodari, C., \& Larrañaga, A. (2015). Airline customer loyalty through analysis of started preference. The Journal of Transport Literature, 9(2), 25-29. https://doi. org/10.1590/2238-1031.jtl.v9n2a5

Maynez, A., Gómez, K., Cavazos, J., Vargas, M., \& Pastrana, M. (2020). Lealtad e intenciones de comportamiento: análisis de diferencias entre pasajeros de aerolíneas mexicanas de bajo costo y de servicio completo. El Periplo Sustentable, 39, 240-263. Recuperado de https:// rperiplo.uaemex.mx/article/view/12035 
Medina, J., Manzanilla, L., \& Díaz, A. (2012). La medición de datos cualitativos, una tendencia en investigación social. Revista Ra Ximbai, 8(2), 287-295. Recuperado de http://www. redalyc.org/pdf/461/46125172004.pdf

Mendoza, C. (2019). Las estrategias de low cost y su relación con la satisfacción del cliente en la aerolínea Latam-Trujillo en el año 2018 (Tesis de maestría, Universidad Privada del Norte, Trujillo, Perú). Recuperado de https://repositorio.upn.edu.pe/handle/11537/23798

Molina, C. (2019). Las aerolíneas tradicionales ganan la batalla a las de bajo costo en 2019. El País. Recuperado de https:/cincodias.elpais.com/cincodias/2019/08/20/companias/ 1566310961_449071.html

Moliner, B., \& Berenguer, G. (2011). El efecto de la satisfacción del cliente en la lealtad: aplicación en establecimientos minoristas. Revista Cuadernos de Administración, 24(42), 101-124. Recuperado de https://www.academia.edu/25982073/ARTICUlO_SATISFACCION_Y_ LEALTAD_DEL_CLIENTE

OACI. (2015). El transporte internacional de la carga aérea. Recuperado de https://www.icao. int/Security/aircargo/Moving\%20Air\%20Cargo\%20Globally/ICAO_WCO_Moving_Air_Cargo_ es.pdf

Oliver, R. L. (1999). Whence consumer loyalty? Journal of Marketing, 63(4-Suppl. 1), 33-44. https://doi.org/10.1177/00222429990634s105

Parra, L., \& Mateus, R. (2020). Análisis comparativo de las aerolíneas bajo costo (modelos nacionales vs. modelos internacionales). Revista Turpade, (12). Recuperado de http://www. turpade.com/noticia.asp? $\mathrm{r}=21 \&$ IDArticulo=89\&IDEdicion $=21 \& \mathrm{~s}=$

Pastrana-Martínez, M., Máynez-Guaderrama, A. I., Vargas-Salgado, M. M., \& Gómez-Bull, K. G. (2020). Análisis de la satisfacción de pasajeros de aerolíneas de bajo costo mexicanas. Estudios Gerenciales, 36(157), 484-495. https://doi.org/10.18046/j.estger.2020.157.3860

Plummer, R. (2015). Cómo responden las aerolíneas a la guerra de precios en el aire. $B B C$ News. Recuperado de https://www.bbc.com/mundo/noticias/2015/11/151105_finde_ economia_aerolineas_tradicionales_bajo_costo_ac

Rodríguez, C. (2018). ¿Cuáles son las diferencias reales entre aerolíneas low cost y regulares? Recuperado de https://www.skyscanner.es/noticias/diferencias-reales-entreaerolineas-low-cost-y-regulares

Sánchez, M. (2009). Volando alto en medio de la turbulencia: Continental Airlines (Tesis de maestría, Universidad Iberoamericana, Ciudad de México, México). Recuperado de http:// 192.203.177.185/bitstream/handle/ibero/1894/015113s.pdf?sequence=1\&isAllowed=y

Setó, D. (2004). De la calidad de servicio a la fidelidad del cliente. Madrid: EsIC Editorial. 
Sheth, J., Allvine, F., Uslay, C., \& Dixit, A. (2007). Deregulation and competition: lessons from the airlines industry. London: SAGE Publications India Pvt Ltd. http://dx.doi. org/10.4135/9788132101598

Singh, J., \& Sirdeshmukh, D. (2000). Agency and trust mechanisms in consumer satisfaction and loyalty judgments. Journal of the Academy of Marketing Science, 28(1), 150-167. https://doi.org/10.1177/0092070300281014

Spreng, R. A., MacKenzie, S. B., \& Olshavsky, R. W. (1996). A reexamination of the determinants of consumer satisfaction. Journal of Marketing, 6O(3), 15-32. https://doi. org/10.1177/002224299606000302

Szymanski, D. M., \& Henard, D. H. (2001). Customer satisfaction: a meta-analysis of the empirical evidence. Journal of the Academy of Marketing Science, 29(1), 16-35. Available from https://link.springer.com/article/10.1177/0092070301291002

Thompson, I. (2005). La satisfacción del cliente. Recuperado de https://www.procase.cl/ Demos/tmk/docs/Satisfacci\%C3\%B3n\%20al\%20Cliente.pdf

Tipiani, F. (2019). Diferencia entre aerolinea tradicional y una low cost. Recuperado de http://infodestino.com/index.php/ultimas-noticias/204-diferencia-entre-aerolinea-tradicional-y-una-low-cost

Torres, M., Paz, K., \& Salazar, F. G. (2019). Métodos de recolección de datos para una investigación. Boletín Electrónico, (3). Recuperado de http://fgsalazar.net/LANDIVAR/ING-PRIMERO/ boletin03/URL_03_BAS01.pdf

Yu, Y. T., \& Dean, A. (2001). The contribution of emotional satisfaction to consumer loyalty. International Journal of Service Industry Management, 12(3), 234-250.

Zárraga, L., Molina, V., \& Corona, E. (2018). La satisfacción del cliente basada en la calidad del servicio a través de la eficiencia del personal y eficiencia del servicio: un estudio empírico de la industria restaurantera. Revista RECAI, 18(7), 46-65. Recuperado de https://recai. uaemex.mx/article/view/9268/8377 